\title{
UNCERTAINTY QUANTIFICATION FOR MAXWELL'S EQUATIONS USING STOCHASTIC COLLOCATION AND MODEL ORDER REDUCTION
}

\author{
Peter Benner \& Judith Schneider*
}

\begin{abstract}
Computational Methods in Systems and Control Theory, Max Planck Institute for Dynamics of Complex Technical Systems, Sandtorstr. 1, 39106 Magdeburg, Germany
\end{abstract}

Original Manuscript Submitted: 10/15/2013; Final Draft Received: 01/21/2015

\begin{abstract}
Modeling and simulation are indispensable for the design process of new semiconductor structures. Difficulties arise from shrinking structure sizes, increasing working frequencies, and uncertainties of materials and geometries. Therefore, we consider the time-harmonic Maxwell's equations for the simulation of a coplanar waveguide with uncertain material parameters. To analyze the uncertainty of the system, we use stochastic collocation with Stroud and sparse grid points. The results are compared to a Monte Carlo simulation. Both methods rely on repetitive runs of a deterministic solver. To accelerate this, we compute a reduced model by means of proper orthogonal decomposition to reduce the computational cost. The Monte Carlo simulation and the stochastic collocation are both applied to the full and the reduced model. All results are compared concerning accuracy and computation time.
\end{abstract}

KEY WORDS: uncertainty quantification, material uncertainties, stochastic collocation, sparse grid, electromagnetics, model order reduction

\section{INTRODUCTION}

Nowadays, the design process of semiconductors is unimaginable without simulations of new micro- and nanoscale systems due to the expensive production of prototypes. However, the numerical simulation of systems that result from modeling of microscale structures is computationally demanding. Two aspects make the simulation even more complicated. One is the ongoing miniaturization of the structures, e.g., the technology improved from $0.35 \mu \mathrm{m}$ (Intel Pentium 133) in 1995 to $20 \mathrm{~nm}$ (Intel Core i7) in 2012, in combination with an increasing of the working frequencies from $100 \mathrm{MHz}$ (Intel's P5 Pentium) in 1995 to 4 GHz (AMD FX 8350) in 2012. This implicates a high-density of electric conductors and induces parasitic effects like cross talk. In the past, the so-called partial element equivalent circuit (PEEC) method [1] was used for the numerical modeling of electromagnetic (EM) properties. Using the PEEC method, the problem is converted from the electromagnetic domain to the circuit domain where traditional circuit solvers can be employed to analyze the equivalent circuit. However, at higher frequencies and for complicated geometries and more complex (e.g., inhomogeneous) materials, the PEEC method is not suitable. For such applications, differential field solvers, such as the finite element method (FEM), are more suitable to compute the EM field by solving Maxwell's equations.

In the following, the electric field intensity $\boldsymbol{E}$ and the magnetic field intensity $\boldsymbol{H}$ are described by Maxwell's equations

$$
\begin{gathered}
\partial_{t}(\epsilon \boldsymbol{E})=\nabla \times \boldsymbol{H}-\sigma \boldsymbol{E}-\boldsymbol{J}, \\
\partial_{t}(\mu \boldsymbol{H})=-\nabla \times \boldsymbol{E}, \\
\nabla \cdot(\epsilon \boldsymbol{E})=\rho, \\
\nabla \cdot(\mu \boldsymbol{H})=0,
\end{gathered}
$$

\footnotetext{
${ }^{*}$ Correspond to Judith Schneider, E-mail: judith.schneider@mpi-magdeburg.mpg.de
} 
where $\rho$ is the charge density and $\boldsymbol{J}$ is the impressed current source. Furthermore, $\epsilon=\epsilon_{r} \cdot \epsilon_{0}$ (permittivity), $\mu=\mu_{r} \cdot \mu_{0}$ (permeability), and $\sigma$ (electrical conductivity) are material-dependent parameters. The equations are considered in a domain $G \subset \mathbb{R}^{3}$.

We decouple the full Maxwell's equations by exploiting the fact that $\nabla \times(\nabla \varphi)=0$ for scalar potentials $\varphi$ and $\nabla \cdot\left(\nabla \times \boldsymbol{A}^{*}\right)=0$ for vector potentials $\boldsymbol{A}^{*}$. Then, (4) yields

$$
\exists \boldsymbol{A}^{*}: \nabla \times \boldsymbol{A}^{*}=\mu \boldsymbol{H} .
$$

Substituting into (2) leads to

$$
\nabla \times\left(\boldsymbol{E}+\partial_{t}\left(\boldsymbol{A}^{*}\right)\right)=0,
$$

which implies the existence of a scalar potential $\varphi$ such that

$$
\boldsymbol{E}=-\nabla \varphi-\partial_{t}\left(\boldsymbol{A}^{*}\right) .
$$

By choosing $\boldsymbol{A}=\boldsymbol{A}^{*}+\int_{t_{0}}^{t} \nabla \varphi d t$ [2], we obtain $\boldsymbol{E}=-\partial_{t}(\boldsymbol{A}), \nabla \times \boldsymbol{A}=\nabla \times \boldsymbol{A}^{*}$, and the vector potential formulation of Maxwell's equations (1)-(4)

$$
\nabla \times\left(\mu^{-1} \nabla \times \boldsymbol{A}\right)+\sigma \partial_{t}(\boldsymbol{A})+\epsilon \partial_{t}^{2}(\boldsymbol{A})=\boldsymbol{J} .
$$

For a further simplification, we work with the time-harmonic form, i.e., we assume $\boldsymbol{A}$ to be given in the form $\boldsymbol{A}=\hat{\boldsymbol{A}} e^{i f t}$, where $f$ is the working frequency and $i=\sqrt{-1}$ the imaginary unit. With this assumption, the time derivatives simplify to

$$
\partial_{t}(\boldsymbol{A})=\text { if } \boldsymbol{A}, \quad \partial_{t}^{2}(\boldsymbol{A})=-f^{2} \boldsymbol{A} .
$$

Therefore, the vector potential formulation can be written as

$$
\nabla \times\left(\mu^{-1} \nabla \times \boldsymbol{A}\right)+i f \sigma \boldsymbol{A}-f^{2} \epsilon \boldsymbol{A}=\boldsymbol{J} .
$$

By replacing $\boldsymbol{E}=-\partial_{t}(\boldsymbol{A})=-$ if $\boldsymbol{A}[3,4]$, we obtain the time-harmonic Maxwell's equation

$$
\nabla \times\left(\mu^{-1} \nabla \times \boldsymbol{E}\right)+i f \sigma \boldsymbol{E}-f^{2} \in \boldsymbol{E}=-i f \boldsymbol{J},
$$

for which the solution $\boldsymbol{E}$ lives in the space $X=\left\{\boldsymbol{E} \in H_{\text {curl }}^{0}(G) \mid \nabla \cdot(\epsilon \boldsymbol{E})=\rho\right\}$, where

$$
H_{\text {curl }}^{0}(G):=\left\{\boldsymbol{E} \in\left(L_{2}(G)\right)^{3} \mid \nabla \times \boldsymbol{E} \in\left(L_{2}(G)\right)^{3}, \boldsymbol{E} \times \boldsymbol{n}=0 \text { on } \partial G\right\}
$$

and

$$
L_{2}(G)=\left\{u \mid \int_{G} u^{2} d \boldsymbol{x}<\infty\right\}
$$

When using the time-harmonic form, we work with a complex electric field. Actually, electric fields are real. Using complex exponentials bears no advantage other than convenient calculation. The imaginary part of the electric field is just a phase shift of the real part. Often the imaginary part is neglected after the computations. We will not neglect it, but work with the absolute value of the electric field.

For the simulation, (5) is discretized by means of the finite element method. This will be further explained in Section 5 .

To allow for fluctuations in the processed materials, the material parameters are treated as uncertain parameters of the system. In this paper, these uncertain parameters are $\epsilon_{r}, \mu_{r}$, and $\sigma$. Therefore, $\boldsymbol{E}$ is parameter dependent but, for reasons of simplification, we will not write this dependence explicitly.

Inaccuracies during the lithography, which lead to variations of the feature structure sizes, lead to another aspect that can no longer be neglected during the simulation. These geometric parameters will not be considered in this work. This aspect will be treated in the future.

For a variational analysis of the effect of uncertainties on the electromagnetic field, methods for uncertainty quantification (UQ) $[5,6]$ are required. The existing methods in this field can be divided into intrusive and nonintrusive 
methods. The motivation for nonintrusive methods is that, in the cooperation with industrial partners the discretization of the system equations is often done by a commercial tool and therefore must be considered as a black box. In this case, intrusive UQ methods, such as stochastic finite elements [7], which would lead to a new discretization respecting the uncertainty in the system, cannot be used. Therefore, we will employ nonintrusive approaches in order to design an algorithm that allows the use of EM field solvers for deterministic problems without accessing the source code. Possible nonintrusive methods are the well-known Monte Carlo (MC) simulation [8, 9], which yields arbitrary exactness but has a slow convergence, or the stochastic collocation approach [10-13]. In our case, the results computed by MC serve as reference solutions. Considering stochastic collocation, the choice of collocation points is very important for the accuracy of the results on the one hand and the effectiveness of the method on the other hand. This will be further investigated in this work.

Another way of saving computation time is to replace the high dimensional discretized system obtained from (5) by a system of reduced order. Such systems can be computed by model order reduction (MOR) [14, 15]. We use a reduced-order model (ROM) instead of the full-order model (FOM) for the MC and the collocation approach. This ROM is obtained by MOR via proper orthogonal decomposition (POD) [16-22].

In the last several years, UQ became more and more popular for solving problems from different application areas. Stroud-based collocation has been used for the statistical characterization of coupled voltages in [11]. In [12], sparse collocation methods with diverse kinds of sample points are applied to electromagnetic scattering by a twodimensional cylinder with an uncertain number of wholes with uncertain size and location. The two choices of sample points, Stroud and sparse grids, are compared in [13] considering as example an elliptic equation. It must be mentioned that Stroud rules exist just for some specific random distributions and are therefore not generally applicable.

Here, we combine components of the latter papers. We use Stroud points [23] and sparse grids [24] and apply the stochastic collocation to the time-harmonic Maxwell's equations in high-frequency range with uncertain material parameters. The use of nonintrusive methods is driven by the application described in Section 2.

The combination of MOR and UQ is rarely studied up to now. One of the first publications considering UQ and MOR for microelectronics is [25], where a projection-based MOR method for variational analysis of RLC interconnect circuits was presented. In [26], a projection based reduction of the state space and a reduction of the random space are applied to an electric network with uncertain capacitances, inductances, and conductances. Another example is [27], where a combination of the reduced basis method and stochastic collocation is applied to stochastic versions of the diffusion equation and the incompressible Navier-Stokes equations.

In this work, we place value on reducing a parameter-affine system once, followed by a repetitive usage of the obtained ROM in the UQ method. The paper is structured as follows. We describe our model application, a coplanar waveguide with dielectric overlay, in Section 2. The UQ methods of interest, Monte Carlo and stochastic collocation, as well as our different choices of collocation points are explained in Section 3. We will give a short introduction to MOR by POD in Section 4 and show the numerical results in Section 5. A short conclusion is given in Section 6.

\section{APPLICATION: A COPLANAR WAVEGUIDE}

The application of interest is a coplanar waveguide with dielectric overlay which is shown in Fig. 1. A detailed description of the coplanar waveguide (only with geometric parameters) can be found in the MOR wiki [28]. The model consists of three perfectly conducting striplines situated at a height of $10 \mathrm{~mm}$ in a shielded box with perfect electric conductor (PEC) boundary. The system is excited at discrete port 1, and the voltage along port 2 is taken as output.

Below a height of $15 \mathrm{~mm}$, there is a substrate, the rest of the box is filled with air. The uncertainties of the model are the material parameters $\epsilon_{r}, \mu_{r}$, and $\sigma$. As the relative permittivity $\epsilon_{r}$ and the conductivity $\sigma$ have different means for substrate and air, we have to work with two sub-domains $G_{s}$ (substrate) and $G_{a}$ (air). That means, we split $\epsilon_{r}$ and $\sigma$ and treat the system as a system with five uncertain parameters.

Due to physical reasons, the parameters must be positive. Therefore, they are assumed to be log-normally distributed. The given means $\mathbb{E}\left(\boldsymbol{p}_{j}\right)$, standard deviations $\operatorname{std}\left(\boldsymbol{p}_{j}\right)$, and the arguments $\boldsymbol{\mu}_{j}$ and $\boldsymbol{\sigma}_{j}$ for the log-normal distributions $\mathcal{L} \mathcal{N}\left(\boldsymbol{\mu}_{j}, \boldsymbol{\sigma}_{j}^{2}\right)$ are given in Table 1 , for $j=1, \ldots, 5$. The assumed probability density function for parameter $\boldsymbol{p}_{j}$ is 


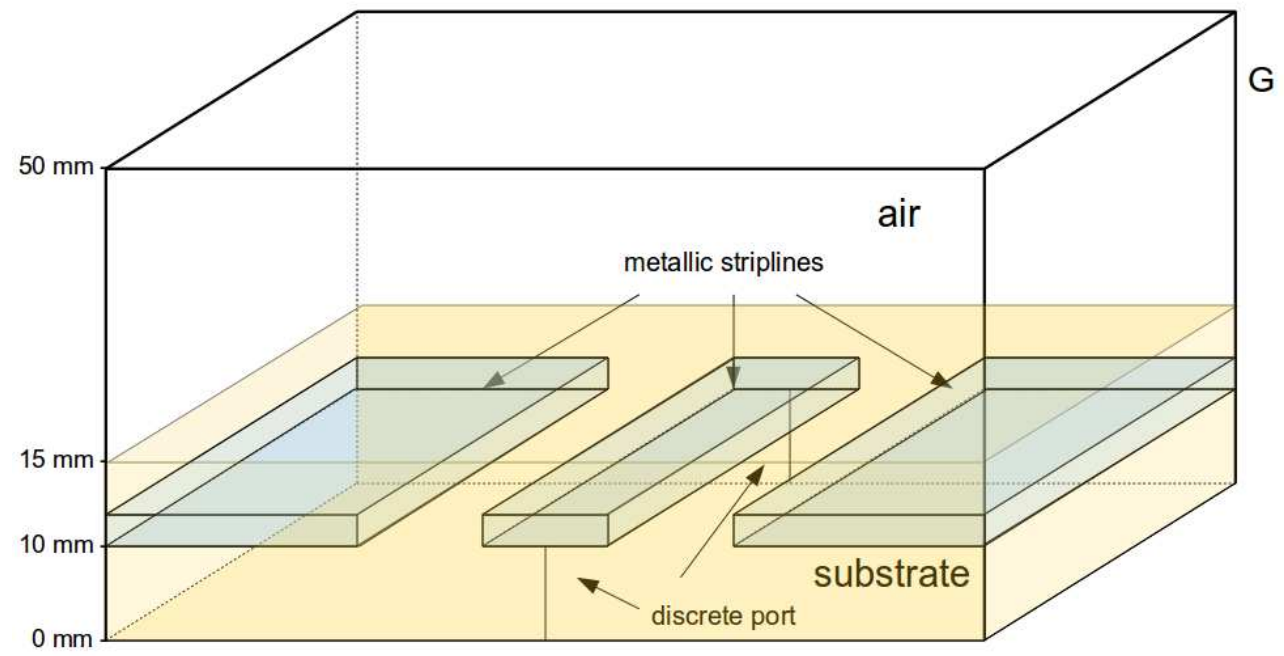

FIG. 1: Coplanar waveguide.

$$
f_{\boldsymbol{p}_{j}}(x):=\frac{1}{\sqrt{2 \pi} \boldsymbol{\sigma}_{j} x} \exp \left(-\frac{\left(\ln (x)-\boldsymbol{\mu}_{j}\right)^{2}}{2 \boldsymbol{\sigma}_{j}^{2}}\right), \quad \text { for } x \in \mathbb{R}, x>0 .
$$

We use the vectors $\boldsymbol{\mu}$ and $\boldsymbol{\sigma}$ to compute the Stroud and sparse grid points. Then we take the exponential of them as sample points, because the exponential of a normally distributed random variable is log-normally distributed. The time-harmonic Maxwell's equations (5) depending on the five parameters listed in Table 1, can be written as follows:

$$
\nabla \times\left(\left(\mu_{r} \mu_{0}\right)^{-1} \nabla \times \boldsymbol{E}\right)+i f\left(\sigma^{s} \mathbb{1}_{G_{s}}+\sigma^{a} \mathbb{1}_{G_{a}}\right) \boldsymbol{E}-f^{2} \epsilon_{0}\left(\epsilon_{r}^{s} \mathbb{1}_{G_{s}}+\epsilon_{r}^{a} \mathbb{1}_{G_{a}}\right) \boldsymbol{E}=-i f \boldsymbol{J},
$$

where $\mathbb{1}_{G_{s}}, \mathbb{1}_{G_{a}}$ denote the indicator functions of sub-domain $G_{s}, G_{a}$, respectively.

In Section 3, we explain the stochastic background and describe different choices of collocation points and their usage for the coplanar waveguide.

\section{NONINTRUSIVE UNCERTAINTY QUANTIFICATION}

Let $(\Omega, \mathcal{F}, \mathcal{P})$ be a probability space, where $\Omega$ is the set of all elementary events, $\mathcal{F}$ is a $\sigma$-algebra of subsets of $\Omega$, and $\mathcal{P}$ is a probability measure on $\mathcal{F}$. Given a vector of independent square integrable random variables $\mathbf{Y}: \Omega \rightarrow \Gamma \subset \mathbb{R}^{N}$, with joint probability density function $f_{\mathbf{Y}}$ and a measurable, possibly vector-valued, function $\boldsymbol{g}: \mathbb{R}^{N} \rightarrow \mathbb{C}^{d}$ for a natural number $d$, we are interested in the computation of statistical quantities like the mean

$$
\mathbb{E}(\boldsymbol{g}(\mathbf{Y})):=\left(\mathbb{E}\left(\boldsymbol{g}_{1}(\mathbf{Y})\right), \ldots, \mathbb{E}\left(\boldsymbol{g}_{d}(\mathbf{Y})\right)\right)^{T},
$$

TABLE 1: Information about the parameter vector $\boldsymbol{p}=\left(\epsilon_{r}^{s}, \epsilon_{r}^{a}, \mu_{r}, \sigma^{s}, \sigma^{a}\right)^{T}$ for the coplanar waveguide

\begin{tabular}{|c|c|c|c|c|c|}
\hline$j$ & $\boldsymbol{p}_{j}$ & $\mathbb{E}\left(\boldsymbol{p}_{j}\right)$ & $s t d\left(\boldsymbol{p}_{j}\right)$ & $\boldsymbol{\mu}_{j}$ & $\boldsymbol{\sigma}_{j}$ \\
\hline 1 & $\epsilon_{r}^{s}$ & 4.40 & $10^{-2}$ & 1.4816 & 0.0023 \\
2 & $\epsilon_{r}^{a}$ & 1.07 & $10^{-2}$ & 0.0676 & 0.0093 \\
3 & $\mu_{r}$ & 1.00 & $10^{-2}$ & 0.0000 & 0.0100 \\
4 & $\sigma^{s}$ & 0.02 & $10^{-4}$ & -3.9120 & 0.0050 \\
5 & $\sigma^{a}$ & 0.01 & $10^{-4}$ & -4.6052 & 0.0100 \\
\hline
\end{tabular}


with

$$
\mathbb{E}\left(\boldsymbol{g}_{k}(\mathbf{Y})\right):=\int_{\Omega} \boldsymbol{g}_{k}(\mathbf{Y}(\boldsymbol{\omega})) d \mathcal{P}(\boldsymbol{\omega}), \quad \text { for } k=1, \ldots, d
$$

and the standard deviation

$$
\operatorname{std}(\mathbf{g}(\mathbf{Y})):=\left(\operatorname{std}\left(\boldsymbol{g}_{1}(\mathbf{Y})\right), \ldots, \operatorname{std}\left(\boldsymbol{g}_{d}(\mathbf{Y})\right)\right)^{T}
$$

with

$$
\operatorname{std}\left(\boldsymbol{g}_{k}(\mathbf{Y})\right):=\sqrt{\mathbb{E}\left(\left|\boldsymbol{g}_{k}(\mathbf{Y})\right|^{2}\right)-\left(\left|\mathbb{E}\left(\boldsymbol{g}_{k}(\mathbf{Y})\right)\right|\right)^{2}}, \quad \text { for } k=1, \ldots, d
$$

of the complex-valued random variable $\boldsymbol{g}(\mathbf{Y})$, see [29]. Here, $|\boldsymbol{g}(\mathbf{Y})|=\sqrt{\operatorname{Re}(\boldsymbol{g}(\mathbf{Y}))^{2}+\operatorname{Im}(\boldsymbol{g}(\mathbf{Y}))^{2}}$ denotes the absolute value of $\boldsymbol{g}(\mathbf{Y})$, which is needed to achieve a real-valued standard deviation. Another reason for the use of this formula for the standard deviation is that, in the end, we are interested in the behavior of the absolute value of $\boldsymbol{g}(\mathbf{Y})$. Therefore, it is important that the standard deviation, which describes the variation of the random vector $\boldsymbol{g}(\mathbf{Y})$ around its mean, is also influenced by the absolute value.

For practical computation, a numerical approximation of (8) and (9) is needed. Here, we use nonintrusive methods that are sampling techniques relying on repetitive runs of a discrete solver.

\subsection{Monte Carlo}

The most popular UQ method is the MC simulation $[8,9]$. The idea behind MC is the law of large numbers, which describes the result of running the same experiment a large number of times. Given a realization $\left(\boldsymbol{\xi}^{1}, \ldots, \boldsymbol{\xi}^{n}\right)$ of a sample $\left(\mathbf{Y}^{1}, \ldots, \mathbf{Y}^{n}\right)$ of the random vector $\mathbf{Y}$, the sample mean of $\boldsymbol{g}(\mathbf{Y})$ is defined by

$$
m_{n}:=\frac{1}{n} \sum_{i=1}^{n} \boldsymbol{g}\left(\boldsymbol{\xi}^{i}\right) .
$$

By the law of large numbers, we have $m_{n} \rightarrow \mathbb{E}(\boldsymbol{g}(\mathbf{Y}))$, for $n \rightarrow \infty$. Therefore, MC uses a randomly chosen realization $\left(\boldsymbol{\xi}^{1}, \ldots, \boldsymbol{\xi}^{n}\right)$ and equal weights for the approximation of $\mathbb{E}(\boldsymbol{g}(\mathbf{Y}))$

$$
\mathbb{E}(\boldsymbol{g}(\mathbf{Y})) \approx m_{n}=\frac{1}{n} \sum_{i=1}^{n} \boldsymbol{g}\left(\boldsymbol{\xi}^{i}\right), \quad \text { for large } n .
$$

The convergence is proportional to $1 / \sqrt{n}$. Considering the coplanar waveguide in Section 2 , the random vector $\mathbf{Y}$ is the vector of uncertain parameters $\boldsymbol{p}$, a five-dimensional vector of log-normally distributed random variables, and $\boldsymbol{E}$ is the function $g$ in which we are interested.

\subsection{Stochastic Collocation}

Another approach based on sampling is the stochastic collocation method. Its effectiveness depends strongly on the choice of collocation points. The idea is to approximate statistical quantities like the mean (7), by an (efficient) quadrature rule

$$
\mathbb{E}(\boldsymbol{g}(\mathbf{Y}))=\int_{\Gamma} \boldsymbol{g}(x) f_{\mathbf{Y}}(x) d x \approx \sum_{i=1}^{n} \boldsymbol{g}\left(\boldsymbol{\xi}^{i}\right) w_{i}=: \hat{\mathbb{E}}(\boldsymbol{g}(\mathbf{Y}))
$$

Here, the realization $\left(\boldsymbol{\xi}^{1}, \ldots, \boldsymbol{\xi}^{n}\right)$, later called the sample points $\left\{\boldsymbol{\xi}^{i}\right\}_{i=1}^{n}$, and the weights $\left\{w_{i}\right\}_{i=1}^{n}$ are determined by use of the probability density function $f_{\mathbf{Y}}$. Higher moments like the standard deviation can be approximated by use of $\hat{\mathbb{E}}(\boldsymbol{g}(\mathbf{Y}))$

$$
\operatorname{std}(\boldsymbol{g}(\mathbf{Y})) \approx \sqrt{\sum_{i=1}^{n}\left|\boldsymbol{g}\left(\boldsymbol{\xi}^{i}\right)\right|^{2} w_{i}-|\hat{\mathbb{E}}(\boldsymbol{g}(\mathbf{Y}))|^{2}}
$$


Like MC, stochastic collocation requires only repetitive runs of an existing deterministic solver. The difference is the choice of sample points and weights. It is always possible to use a tensor product of a one-dimensional quadrature formula. For many parameters, this becomes very expensive. Therefore, we use two other options that suffer less from the curse of dimensionality. One way is to use only a part of the tensor grid, which is done by the Smolyak algorithm. The result is called a sparse grid. Another way to compute the points are the Stroud integration rules, which yield a very small number of points and have a fixed accuracy. Both options are described below.

\subsubsection{Stroud-3 Integration Rules}

The Stroud-3 integration rule was introduced in 1957 by Arthur Stroud [23] and is accurate for polynomials up to degree 3. It yields either beta or normally distributed points, which are weighted by $1 / n$, where $n$ is the number of points. Besides that, there are the Stroud-2 rule for beta, gamma, or normally distributed points and several Stroud rules with varying accuracy for the uniform distribution. Stroud published his vast collection of quadrature formulas in 1971 [30]. In our case only the normally distributed Stroud-3 points are considered. This choice is motivated by the physics of the considered application (i.e., the fact that the parameters are assumed to be log-normally distributed) as explained in Section 2.

For a system with $N$ parameters only $2 N$ sample points are needed. The $j$ th component of the normally distributed points with mean $\boldsymbol{\mu}_{j}$ and standard deviation $\boldsymbol{\sigma}_{j}$ is given in [11] as follows:

$$
x_{j}^{i}=\boldsymbol{\sigma}_{j} z_{j}^{i}+\boldsymbol{\mu}_{j},
$$

where for $i=2 r-1, i=2 r$, respectively, and $r=1,2, \ldots,\lfloor N / 2\rfloor$,

$$
z_{j}^{2 r-1}=\sqrt{2} \cos \left(\frac{(2 r-1) j \pi}{N}\right), \quad \quad z_{j}^{2 r}=\sqrt{2} \sin \left(\frac{(2 r-1) j \pi}{N}\right) .
$$

If $N$ is odd, then $z_{j}^{N}=(-1)^{j}$. Here, $\lfloor N / 2\rfloor$ is the biggest integer number smaller or equal than $N / 2$.

Stroud-based stochastic collocation has been discussed in $[11,13]$. The used Stroud-3 rule is very efficient, especially for systems with many parameters, because of the very small number of sample points. Unfortunately, the accuracy is fixed and there are no higher order Stroud rules for the normal distribution. Figure 2(a) shows the standard normally distributed Stroud points for a system with two parameters. In the case of the coplanar waveguide, Stroud yields $n=10$ sample points $\left\{\boldsymbol{\xi}^{i}\right\}_{i=1}^{10}$ in the five-dimensional parameter space.

\subsubsection{Hermite Genz-Keister Sparse Grids}

Sparse grids are constructed by the Smolyak algorithm [31, 32] which is a linear combination of product formulas. Every one-dimensional quadrature rule can be chosen and the linear combination preserves the interpolation properties

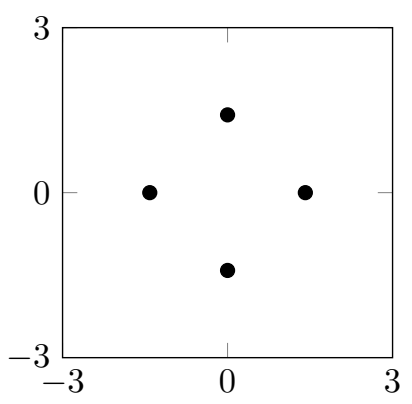

(a) Stroud

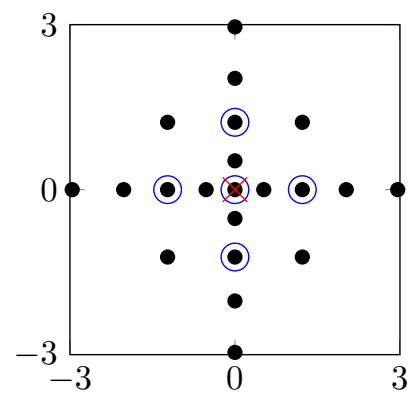

(b) HGK sparse grids levels 0-2

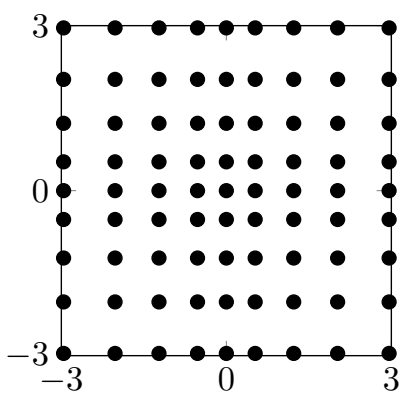

(c) HGK tensor grid

FIG. 2: Comparison of Stroud, HGK sparse grids of level 0 (x), 1 (circles) and 2 (dots), and HGK tensor grid in two dimensions. 
of the univariate case for higher dimensions. We use a one-dimensional Hermite Genz-Keister (HGK) rule [33, 34] as starting point for the Smolyak algorithm. Genz and Keister [33] developed multidimensional Gauss-Hermite (GH) [35] schemes that have several nice features. As Gauss-Patterson (GP) rules [12, 36, 37] they are nested but in contrast to them, they are computed on infinite regions. Furthermore, for a fixed order, the number of grid points growths moderate exponentially, as is the case for Clenshaw-Curtis and GP. Moderate exponential growth means that we select the smallest exponential order of the sequence of selected rules so that $4 L+1$ is smaller or equal to the desired precision, where $L$ is the index of the selected rules. As an example, for a desired precision of 5 we need $L \leq 1$, which leads to the order $2 L+1=3$. The advantage compared to a usual GH rule is the nestedness.

Because the fact that they are computed using a quite involved scheme, HGK rules do not exist for arbitrary numbers of generating points. The beginning is a one-point Gauss-Hermite rule, which has degree 1. By adding new generators to that rule, we can achieve rules with degree $3,15,19,29,51,63$, and 67 [33]. The higher the level of the sparse grid is, the higher the number of generating points of the underlying one-dimensional quadrature rule.

To illustrate the sparsity of sparse grids and to show the nestedness of HGK sparse grids, Figure 2(b) shows the HGK sparse grids of levels 0,1 , and 2 (HGK 0,1, and 2) with $n=1,5$, and 21 grid points, respectively. Figure 2(c) shows the corresponding tensor grid with $n=81$ grid points for a standard normally distributed variable in two dimensions. In case of the coplanar waveguide, we use HGK sparse grids of levels 0,1 , and 2 in the fivedimensional parameter space, which have 1,11 , and 81 points and are accurate for polynomials up to degree 1,5 , and 15 , respectively. Certainly, the number of system evaluations needed for our example is rather small. But the described collocation can also be used for higher dimensional problems with a higher number of parameters, where much more evaluations of the system are needed.

In Section 4, we explain a POD-based MOR method that combined with either MC or sparse grid interpolation can reduce the computational effort.

\section{MODEL ORDER REDUCTION}

UQ via MC or stochastic collocation requires numerous full-order EM field solves which can be a time-consuming task for complicated 3D geometries. It is thus our goal to combine this approach with MOR for the time-harmonic Maxwell's equations (6) to reduce the computational cost. We need a ROM that preserves the statistical properties of the FOM.

In this work, we use a POD-based MOR as described in [19]. POD was first mentioned under this name in [16]. Its central issue is the reduction of data revealing the essential information with the aid of a few basis vectors. We will explain the close connection to the singular value decomposition (SVD) [38] of rectangular matrices in this section.

For a given matrix $Y=\left[y_{1}, \ldots, y_{m_{2}}\right] \in \mathbb{R}^{m_{1} \times m_{2}}$ with rank $d \leq \min \left\{m_{1}, m_{2}\right\}$, the SVD ensures the existence of real numbers $\sigma_{1} \geq \sigma_{2} \geq \ldots \geq \sigma_{d}>0$ and orthogonal matrices $U=\left[u_{1}, \ldots, u_{m_{1}}\right] \in \mathbb{R}^{m_{1} \times m_{1}}$ and $V=$ $\left[v_{1}, \ldots, v_{m_{2}}\right] \in \mathbb{R}^{m_{2} \times m_{2}}$ such that

$$
U^{T} Y V=\left(\begin{array}{cc}
D & 0 \\
0 & 0
\end{array}\right)=: \Sigma \in \mathbb{R}^{m_{1} \times m_{2}}
$$

with $D=\operatorname{diag}\left(\sigma_{1}, \ldots, \sigma_{d}\right) \in \mathbb{R}^{d \times d}$ and zero matrices of appropriate dimensions. For $\ell \in\{1, \ldots, d\}$, the solution to

$$
\max _{\tilde{u}_{1}, \ldots, \tilde{u}_{\ell} \in \mathbb{R}^{m_{1}}} \sum_{i=1}^{\ell} \sum_{j=1}^{m_{2}}\left|\left\langle y_{j}, \tilde{u}_{i}\right\rangle_{\mathbb{R}^{m_{1}}}\right|^{2}, \quad \text { s.t. } \quad\left\langle\tilde{u}_{i}, \tilde{u}_{j}\right\rangle_{\mathbb{R}^{m_{1}}}=\delta_{i j} \quad \text { for } 1 \leq i, j \leq \ell
$$

is given by the first $\ell$ columns of $U,\left\{u_{i}\right\}_{i=1}^{\ell}$, which yields the POD basis of rank $\ell$. For a given $\ell \leq d$, the POD yields the best approximation of the columns of $Y$ among all rank $\ell$ approximations. The vectors $y_{1}, \ldots, y_{m_{2}}$ are called snapshots, a designation that was first used in [17]. The usage of POD for our example is further explained in Section 5 . 


\section{NUMERICAL RESULTS}

We show some numerical results for the coplanar waveguide in Fig. 1, that are achieved by the previously explained collocation method. The discretization of the affine system (6) and the assembling of the matrices [39] is done in FEniCS [40, 41] by use of Nédélec finite elements [42, 43]. In the finite element context, a weak solution of (6) is searched instead of a classical one. Therefore, we employ a variational formulation, i.e., we multiply (6) by a test function $\boldsymbol{v} \in H_{\text {curl }}(G):=\left\{\boldsymbol{v} \in\left(L_{2}(G)\right)^{3} \mid \nabla \times \boldsymbol{v} \in\left(L_{2}(G)\right)^{3}\right\}$ and integrate over $G$. This leads to

$$
\begin{aligned}
\int_{G} \nabla \times\left(\left(\mu_{r} \mu_{0}\right)^{-1} \nabla \times \boldsymbol{E}\right) \boldsymbol{v} d \boldsymbol{x} & +\int_{G} i f\left(\sigma^{s} \mathbb{1}_{G_{s}}+\sigma^{a} \mathbb{1}_{G_{a}}\right) \boldsymbol{E} \boldsymbol{v} d \boldsymbol{x} \\
& -\int_{G} f^{2} \epsilon_{0}\left(\epsilon_{r}^{s} \mathbb{1}_{G_{s}}+\epsilon_{r}^{a_{1}} \mathbb{1}_{G_{a}}\right) \boldsymbol{E} \boldsymbol{v} d \boldsymbol{x}=-\int_{G} i f \boldsymbol{J} \boldsymbol{v} d \boldsymbol{x} .
\end{aligned}
$$

Now the first integral is integrated by parts [44], which leads to

$$
\begin{aligned}
& \int_{G}\left(\left(\mu_{r} \mu_{0}\right)^{-1} \nabla \times \boldsymbol{E}\right)(\nabla \times \boldsymbol{v}) d \boldsymbol{x}-\int_{\partial G}\left(\mu_{r} \mu_{0}\right)^{-1}((\nabla \times \boldsymbol{E}) \times \boldsymbol{n}) \boldsymbol{v} d s \\
& +\int_{G} i f\left(\sigma^{s} \mathbb{1}_{G_{s}}+\sigma^{a} \mathbb{1}_{G_{a}}\right) \boldsymbol{E} \boldsymbol{v} d \boldsymbol{x}-\int_{G} f^{2} \epsilon_{0}\left(\epsilon_{r}^{s} \mathbb{1}_{G_{s}}+\epsilon_{r}^{a} s \mathbb{1}_{G_{a}}\right) \boldsymbol{E} \boldsymbol{v} d \boldsymbol{x}=-\int_{G} i f \boldsymbol{J} \boldsymbol{v} d \boldsymbol{x} .
\end{aligned}
$$

For the boundary integral, it can easily be seen that

$$
\int_{\partial G}\left(\mu_{r} \mu_{0}\right)^{-1}((\nabla \times \boldsymbol{E}) \times \boldsymbol{n}) \boldsymbol{v} d s=\int_{\partial G}\left(\mu_{r} \mu_{0}\right)^{-1}(\nabla \times \boldsymbol{E})(\boldsymbol{n} \times \boldsymbol{v}) d s,
$$

which is equal to zero if $\boldsymbol{n} \times \boldsymbol{v}=0$. Therefore, we obtain the weak formulation

$$
\begin{aligned}
\int_{G}\left(\left(\mu_{r} \mu_{0}\right)^{-1} \nabla \times \boldsymbol{E}\right)(\nabla \times \boldsymbol{v}) d \boldsymbol{x} & +\int_{G} i f\left(\sigma^{s} \mathbb{1}_{G_{s}}+\sigma^{a} \mathbb{1}_{G_{a}}\right) \boldsymbol{E} \boldsymbol{v} d \boldsymbol{x} \\
& -\int_{G} f^{2} \epsilon_{0}\left(\epsilon_{r}^{s} \mathbb{1}_{G_{s}}+\epsilon_{r}^{a_{1}} \mathbb{1}_{G_{a}}\right) \boldsymbol{E} \boldsymbol{v} d \boldsymbol{x}=-\int_{G} i f \boldsymbol{J} \boldsymbol{v} d \boldsymbol{x},
\end{aligned}
$$

for all $v \in H_{\text {curl }}^{0}(G)$.

The system is discretized by replacing the domain $G$ by a finite-dimensional closed subspace, in our case a finite element grid $G_{h}$ with 18755 degrees of freedom (dof). For $\boldsymbol{w}, \boldsymbol{v} \in H_{\text {curl }}^{0}\left(G_{h}\right), \mathbb{1}_{\text {in }}$ and $\mathbb{1}_{\text {out }}$ the indicator function for the input and output region, respectively, and $l=a, s$, we define

$$
\begin{aligned}
A_{\mu_{0}} & =\int_{G_{h}}\left(\mu_{0}^{-1} \nabla \times \boldsymbol{w}\right)(\nabla \times \boldsymbol{v}) d \boldsymbol{x} \\
A_{\epsilon_{0}}^{l} & =\int_{G_{h}} \epsilon_{0} \mathbb{1}_{G_{l}} \boldsymbol{w} \boldsymbol{v} d \boldsymbol{x} \\
A^{l} & =\int_{G_{h}} \mathbb{1}_{G_{l}} \boldsymbol{w} \boldsymbol{v} d \boldsymbol{x} \\
B & =-\int_{G_{h}} i f \mathbb{1}_{\text {in }} \boldsymbol{v} d \boldsymbol{x} \\
C & =\int_{G_{h}} \mathbb{1}_{\text {out }} \boldsymbol{v} d \boldsymbol{x} .
\end{aligned}
$$

Hence, the affine discretized form of (10) is

$$
\begin{aligned}
\mu_{r} A_{\mu_{0}} \boldsymbol{e}(\boldsymbol{p})-f^{2}\left(\epsilon_{r}^{s} A_{\epsilon_{0}}^{s}+\epsilon_{r}^{a} A_{\epsilon_{0}}^{a}\right) \boldsymbol{e}(\boldsymbol{p})+i f\left(\sigma^{s} A^{s}+\sigma^{a} A^{a}\right) \boldsymbol{e}(\boldsymbol{p}) & =B u, \\
y(\boldsymbol{p}) & =C \boldsymbol{e}(\boldsymbol{p}),
\end{aligned}
$$


where the second equation describes the output behavior of the system, $y(\boldsymbol{p})$ is the one-dimensional output voltage, $u$ the input current of 1 Ampère in our example, and $\boldsymbol{e}(\boldsymbol{p})$ the discretized electrical field, living on $G_{h}$. Furthermore, the matrices $A_{\epsilon_{0}}^{a}, A_{\epsilon_{0}}^{s}, A^{a}$, and $A^{s}$ have nonzero entries only on the corresponding subdomain. We consider a working frequency of $f=0.6 \times 10^{9}$. All system evaluations are computed with the direct MATLAB ${ }^{\circledR}$ solver backslash based on the sparse direct solver UMFPACK [45].

We want to approximate the mean and the standard deviation of the solution $\boldsymbol{e}(\boldsymbol{p}) \in H_{\text {curl }}^{0}\left(G_{h}\right)$ and the output $y(\boldsymbol{p})$ of (11) componentwise via

$$
\begin{aligned}
& \mathbb{E}(\boldsymbol{e}(\boldsymbol{p})) \approx \sum_{i=1}^{n} \boldsymbol{e}\left(\boldsymbol{\xi}^{i}\right) w_{i} \\
& \mathbb{E}(y(\boldsymbol{p})) \approx \sum_{i=1}^{n} y\left(\boldsymbol{\xi}^{i}\right) w_{i}
\end{aligned}
$$$$
\begin{aligned}
\operatorname{std}(\boldsymbol{e}(\boldsymbol{p})) & \approx \sqrt{\sum_{i=1}^{n}\left|\boldsymbol{e}\left(\boldsymbol{\xi}^{i}\right)\right|^{2} w_{i}-|\mathbb{E}(\boldsymbol{e}(\boldsymbol{p}))|^{2}}, \\
\operatorname{std}(y(\boldsymbol{p})) & \approx \sqrt{\sum_{i=1}^{n}\left|y\left(\boldsymbol{\xi}^{i}\right)\right|^{2} w_{i}-|\mathbb{E}(y(\boldsymbol{p}))|^{2}},
\end{aligned}
$$

using stochastic collocation with sample points $\left\{\boldsymbol{\xi}^{i}\right\}_{i=1}^{n}$ obtained by Stroud or HGK sparse grids with the corresponding weights $w_{i}$. For reasons of simplification, we omit the $\boldsymbol{p}$ dependence of $\boldsymbol{e}$ and $y$ in the following.

For the approximations computed via $\mathrm{MC}$, i. e. $\mathbb{E}_{M C}(\boldsymbol{e}), \operatorname{std}_{M C}(\boldsymbol{e}), \mathbb{E}_{M C}(y)$, and $s t d_{M C}(y)$, we compare the following errors for different choices of collocation points

$$
\begin{aligned}
\operatorname{err}_{\mathbb{E}(\boldsymbol{e})}^{r e l} & :=\left|\frac{\mathbb{E}(\boldsymbol{e}(\boldsymbol{x}))-\mathbb{E}_{M C}(\boldsymbol{e}(\boldsymbol{x}))}{\mathbb{E}_{M C}(\boldsymbol{e}(\boldsymbol{x}))}\right|, \\
e r r_{s t d(\boldsymbol{e})}^{r e l} & :=\left|\frac{s t d(\boldsymbol{e}(\boldsymbol{x}))-s t d_{M C}(\boldsymbol{e}(\boldsymbol{x}))}{s t d_{M C}(\boldsymbol{e}(\boldsymbol{x}))}\right|, \\
\operatorname{err}_{\mathbb{E}(y)}^{r e l} & :=\left|\frac{\mathbb{E}(y)-\mathbb{E}_{M C}(y)}{\mathbb{E}_{M C}(y)}\right|, \\
e r r_{s t d(y)}^{r e l}(y) & :=\left|\frac{s t d(y)-s t d_{M C}(y)}{s t d_{M C}(y)}\right| .
\end{aligned}
$$

Because the electric field is determined on the whole domain, we need to evaluate it in every dof of the FEM grid and compute the relative error locally. This is expressed by writing $\boldsymbol{e}(\boldsymbol{x})$.

The MC simulation for the reference solution is implemented in MATLAB and operates on one million sample points. The variance of the MC with respect to the number of MC sample points is shown in Fig. 3. It can be seen that the variance for the electric field $\boldsymbol{e}$ is much higher than for the output $y$, but both are converging.

The collocation is also implemented in MATLAB. The HGK sparse grids are computed by means of the MATLAB library SGMGA [46]. Table 2 shows the relative errors for mean and standard deviation of $\boldsymbol{e}$ and $y$.

HGK 0 is not able to approximate the reference solution for the standard deviations since the single grid point is the mean vector of the parameters and hence the standard deviation is zero. On the other hand, we observe that HGK 1 is already sufficient for this example as there is no considerable improvement when we use HGK 2. Note that HGK 2 consists of 81 grid points. Stroud and HGK 1 are comparable concerning complexity and accuracy.

For the POD, we need snapshots of the parameters that are then used to compute snapshots of the electrical field. We have several possibilities for the choice of snapshots. One could use either the Stroud or the HGK sparse grid sample points for the computation of the snapshots of the electrical field. Both choices do not yield useful ROMs.

In our example, we use three snapshots for every single parameter $\boldsymbol{p}_{j}$, namely $\boldsymbol{\mu}_{j}-3 \boldsymbol{\sigma}_{j}, \boldsymbol{\mu}_{j}$, and $\boldsymbol{\mu}_{j}+3 \boldsymbol{\sigma}_{j}$. This choice is motivated by the fact that $99 \%$ of all realizations of a $\mathcal{N}(\mu, \sigma)$-distributed random variable lie in the interval $[\mu-3 \sigma, \mu+3 \sigma]$. That means we reach nearly all possible outcomes and cut the less important tails. Each of the $3^{5}$ parameter snapshots yields a snapshot of the electrical field. These $\boldsymbol{e}$-snapshots are stored in the snapshot matrix $Y$, see Section 4 of which we compute the singular values via the MATLAB function svd

$$
[U, D, V]=\operatorname{svd}(Y, 0)
$$




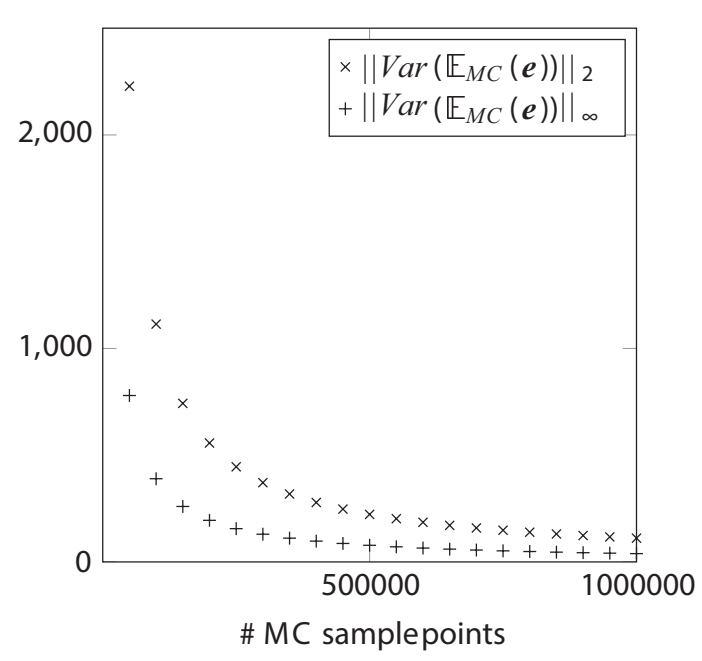

(a) Variance of $\mathbb{E}_{M C}(\boldsymbol{e})$.

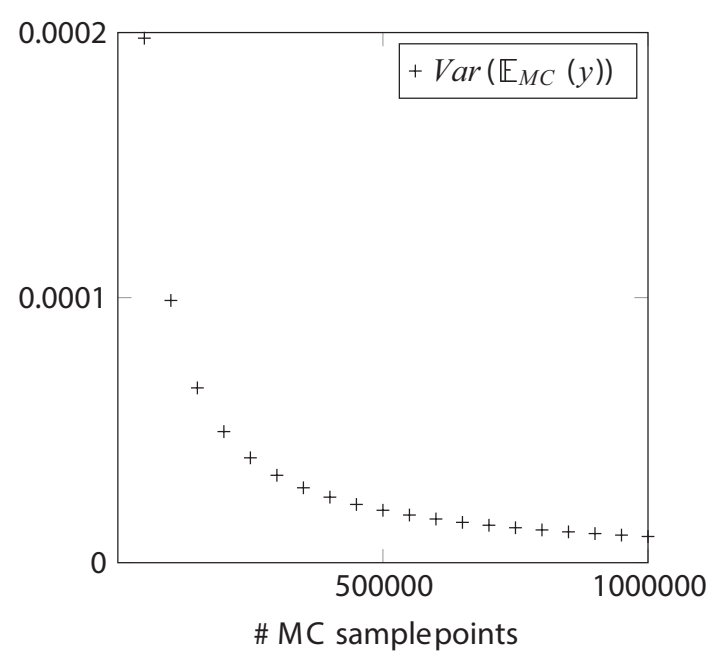

(b) Variance of $\mathbb{E}_{M C}(y)$.

FIG. 3: Monte Carlo convergence.

TABLE 2: Relative errors for the coplanar waveguide

\begin{tabular}{|c|c|c|c|c|c|c|}
\hline Method & $\left\|e r r_{\mathbb{E}(\boldsymbol{e})}^{r e l}\right\|_{2}$ & $\left\|\operatorname{err}_{\mathbb{E}(\boldsymbol{e})}^{r e l}\right\|_{\infty}$ & $\left\|\operatorname{err}_{\text {std }}^{r e l}(\boldsymbol{e})\right\|_{2}$ & $\left\|e r r_{s t d(\boldsymbol{e})}^{r e l}\right\|_{\infty}$ & $e r r_{\mathbb{E}(y)}^{r e l}$ & $e r r_{s t d(y)}^{r e l}$ \\
\hline Stroud & $1.13 \times 10^{-3}$ & $6.69 \times 10^{-5}$ & $7.12 \times 10^{-2}$ & $1.06 \times 10^{-3}$ & $8.76 \times 10^{-6}$ & $4.03 \times 10^{-4}$ \\
\hline HGK 0 & $43 \times 10^{-3}$ & $7.43 \times 10^{-4}$ & - & - & $4.58 \times 10^{-5}$ & - \\
\hline $\mathrm{HGI}$ & $13 \times 10^{-3}$ & $6.69 \times 10^{-5}$ & $7.64 \times 10^{-2}$ & $1.11 \times 10^{-3}$ & $8.76 \times 10^{-6}$ & $4.51 \times 10^{-4}$ \\
\hline HGK 2 & $1.13 \times 10^{-3}$ & $6.69 \times 10^{-5}$ & $7.55 \times 10^{-2}$ & $1.10 \times 10^{-3}$ & $8.76 \times 10^{-6}$ & $4.52 \times 10^{-4}$ \\
\hline $\mathrm{MC}(\mathrm{POD}$ & $.79 \times 10^{-8}$ & $7.35 \times 10^{-8}$ & $1.83 \times 10^{-7}$ & $3.04 \times 10^{-8}$ & $6.96 \times 10^{-12}$ & $4.10 \times 10^{-11}$ \\
\hline Stroud (POD 10) & & $6.69 \times 10^{-5}$ & $7.12 \times 10^{-2}$ & $1.06 \times 10^{-3}$ & $8.76 \times 10^{-6}$ & $4.03 \times 10^{-4}$ \\
\hline HGK 0 (POD 10) & $6.43 \times 10^{-3}$ & $7.43 \times 10^{-4}$ & - & - & $4.58 \times 10^{-5}$ & - \\
\hline HGK 1 (POD 10) & $1.13 \times 10^{-3}$ & $6.69 \times 10^{-5}$ & $7.64 \times 10^{-2}$ & $1.11 \times 10^{-3}$ & $8.76 \times 10^{-6}$ & $4.51 \times 10^{-4}$ \\
\hline HGK 2 (POD 10) & $1.13 \times 10^{-3}$ & $6.69 \times 10^{-5}$ & $7.55 \times 10^{-2}$ & $1.10 \times 10^{-3}$ & $8.76 \times 10^{-6}$ & $4.52 \times 10^{-4}$ \\
\hline
\end{tabular}

As in our case $m_{1}=m_{2}$, we can only use the truncated version of $U, U_{\text {red }} \in \mathbb{R}^{\ell \times m_{1}}$, for the computation of the reduced matrices and vectors and ignore $V$. This leads to

$$
\begin{array}{lll}
A_{j}^{r e d}=U_{r e d}^{T} A_{j} U_{\text {red }}, & \text { for all system matrices } A_{j} \in \mathbb{R}^{m_{1} \times m_{1}} \text { in (11), } \\
B_{j}^{r e d}=U_{r e d}^{T} B_{j}, & \text { for all system vectors } B_{j} \in \mathbb{R}^{m_{1} \times 1} \quad \text { in (11). }
\end{array}
$$

As all the system matrices are parameter independent in our example, we can use the resulting reduced matrices and vectors for all needed system evaluations. The computed electrical field $\boldsymbol{e}_{\text {red }}$ is of reduced dimension, too. It is then prolongated on the full-dimensional grid via

$$
\boldsymbol{e}=U_{\text {red }} \boldsymbol{e}_{\text {red }}
$$

The achieved singular value decay is shown in Fig. 4. We use a POD basis of rank $\ell=10$ (see the horizontal line in Fig. 4), since the ratio of $\sigma_{1}$ and $\sigma_{10}$ is already of order $10^{12}$. Therefore, the reduced model has dimension 10 . The computation of the reduced model took $\sim 4 \mathrm{~min}$. Considering as deterministic model the system evaluated at the mean 


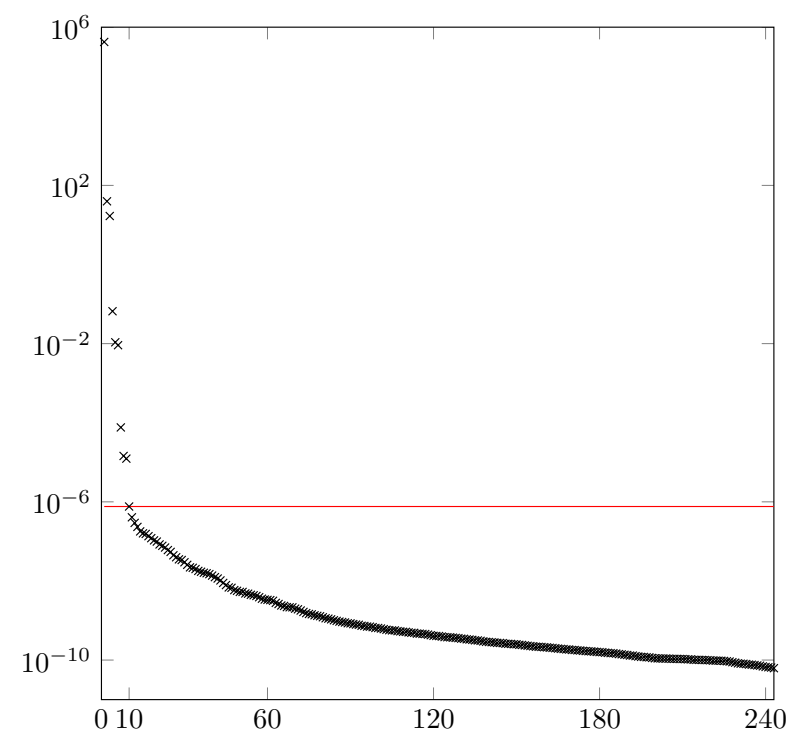

FIG. 4: Singular value decay for POD with $3^{5}$ snapshots.

of the parameters with solution $\boldsymbol{e}_{\text {full }}$ shown in Fig. 5 and $y_{f u l l}$, we can compute the relative errors for the reduced deterministic model with solution $\boldsymbol{e}_{P O D}$ and $y_{P O D}$

$$
\begin{aligned}
\left\|e r r_{\boldsymbol{e}}^{r e l}\right\|_{2} & :=\left\|\frac{\boldsymbol{e}_{\text {full }}(\boldsymbol{x})-\boldsymbol{e}_{P O D}(\boldsymbol{x})}{\boldsymbol{e}_{\text {full }}(\boldsymbol{x})}\right\|_{2}=9.88 \times 10^{-8}, \\
\left\|e r r_{\boldsymbol{e}}^{r e l}\right\|_{\infty} & :=\left\|\frac{\boldsymbol{e}_{\text {full }}(\boldsymbol{x})-\boldsymbol{e}_{P O D}(\boldsymbol{x})}{\boldsymbol{e}_{\text {full }}(\boldsymbol{x})}\right\|_{\infty}=7.36 \times 10^{-8}, \\
e r r_{y}^{r e l} & :=\left|\frac{y_{\text {full }}-y_{P O D}}{y_{\text {full }}}\right|=8.22 \times 10^{-12} .
\end{aligned}
$$

The deterministic errors are small, which explains that the results for the MC simulation of the reduced model (see Table 2) are even better than the collocation results for the full model. Besides that, the collocation for the reduced model is as good as the collocation for the full model.

The computation time needed for the system evaluations on an Intel ${ }^{\circledR}$ Core2 Duo CPU $3 \mathrm{GHz}$ with $4 \mathrm{~GB}$ RAM available is shown in Table 3. These times confirm that the combination of Stroud or sparse grid-based collocation and MOR is a good time-saving alternative for higher dimensional problems. The fact that the errors for the output $y$ are smaller than the ones for the electrical field $\boldsymbol{e}$ for all methods, can be explained by looking at Fig. 6, which shows
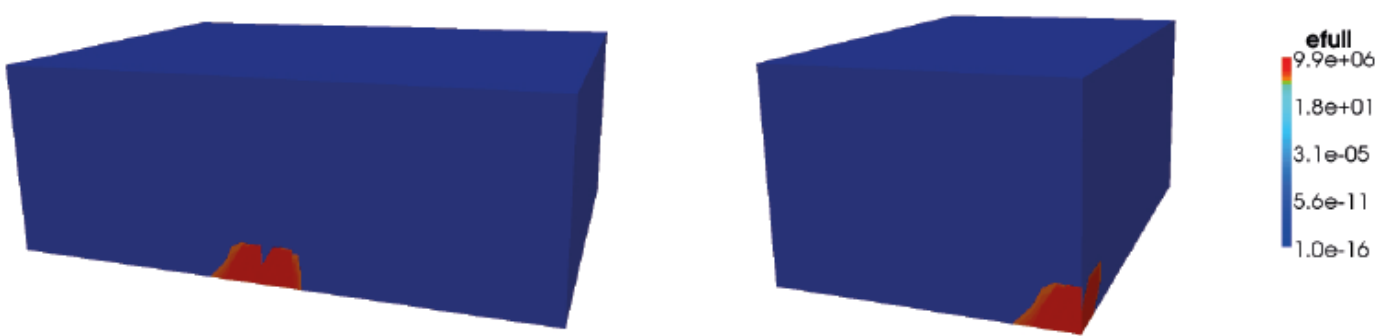

FIG. 5: Absolute value of $\boldsymbol{e}_{\text {full }}$, the solution of the system for the mean of the parameters, plotted in logarithmic scale, shown on the full and on the left half of the domain. 
TABLE 3: Computation time in days:hours:minutes:seconds

\begin{tabular}{|c|c|c|c|c|c|}
\hline Model $\backslash$ Method & Stroud $(n=10)$ & HGK $0(n=1)$ & HGK $1(n=11)$ & HGK $2(n=81)$ & MC $\left(n=10^{6}\right)$ \\
\hline FOM $(18755$ dofs $)$ & $00: 00: 00: 13$ & $00: 00: 00: 05$ & $00: 00: 00: 14$ & $00: 00: 01: 15$ & $10: 06: 58: 34$ \\
ROM $(10$ dofs $)$ & $00: 00: 00: 04$ & $00: 00: 00: 03$ & $00: 00: 00: 04$ & $00: 00: 00: 04$ & $00: 00: 07: 44$ \\
\hline
\end{tabular}
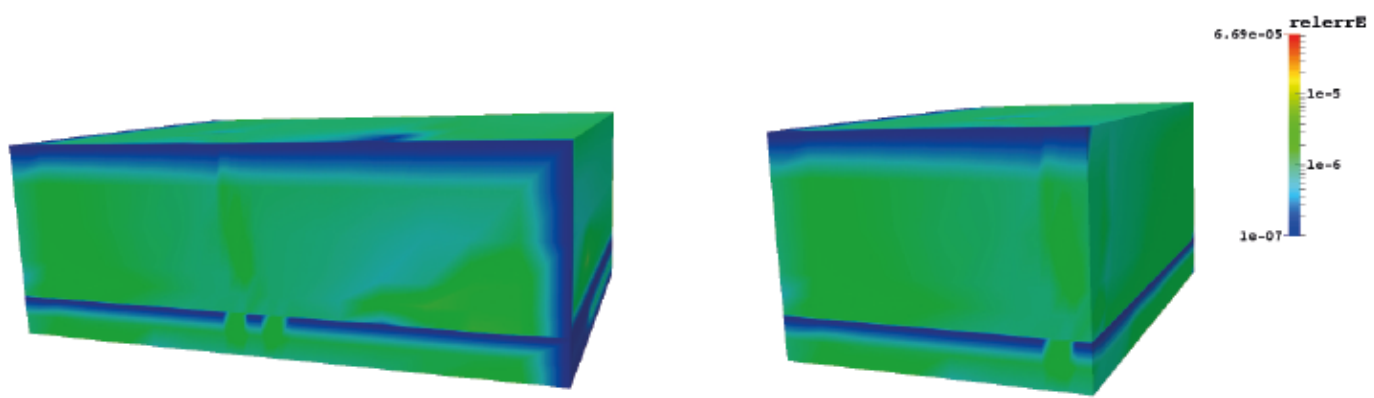

FIG. 6: $\operatorname{err} r_{\mathbb{E}(\boldsymbol{e})}^{r e l}$ for Stroud, plotted in logarithmic scale.

the relative error for the mean of the electric field $\boldsymbol{e}$ computed via Stroud-based collocation on the whole domain $G_{h}$ and on the left half of the domain. There it can be seen that the error is small in the region around the discrete port, which is relevant for the computation of the output $y$. For comparison, we plotted $\operatorname{err}_{\mathbb{E}(\boldsymbol{e})}^{\text {rel }}$ for MC of the reduced model in Fig. 7. The regions where a visible error occurs are smaller. Both Figs. 6 and 7 have a logarithmic scale, and the legends range from the minimum to the maximum of the relative error.

\section{CONCLUDING REMARKS}

In this paper, we described several techniques for UQ of the time-harmonic Maxwell's equations. We showed that stochastic collocation is well suited for the variational analysis of a coplanar waveguide if the collocation points are chosen carefully. Stroud points as well as HGK sparse grid points lead to an efficient computation of the statistical quantities. We compared the results to a very accurate but computationally very costly MC simulation.

On the other hand, we reduced the computation time of the MC simulation by replacing the FOM in the repetitive runs of the deterministic solver by a ROM. This was motivated by the observation that ROMs computed by POD approximate the FOM very well for time-harmonic Maxwell's equations with uncertain parameters with small standard deviations. We achieved a high accuracy for ROMs of very small order. Therefore, the combination of MC and MOR turned out to be a good alternative to stochastic collocation based on the FOM.

We also replaced the FOM by a ROM in the stochastic collocation method. This approach does not gain that much from MOR since POD requires more evaluations of the FOM than the collocation itself. This would be different for
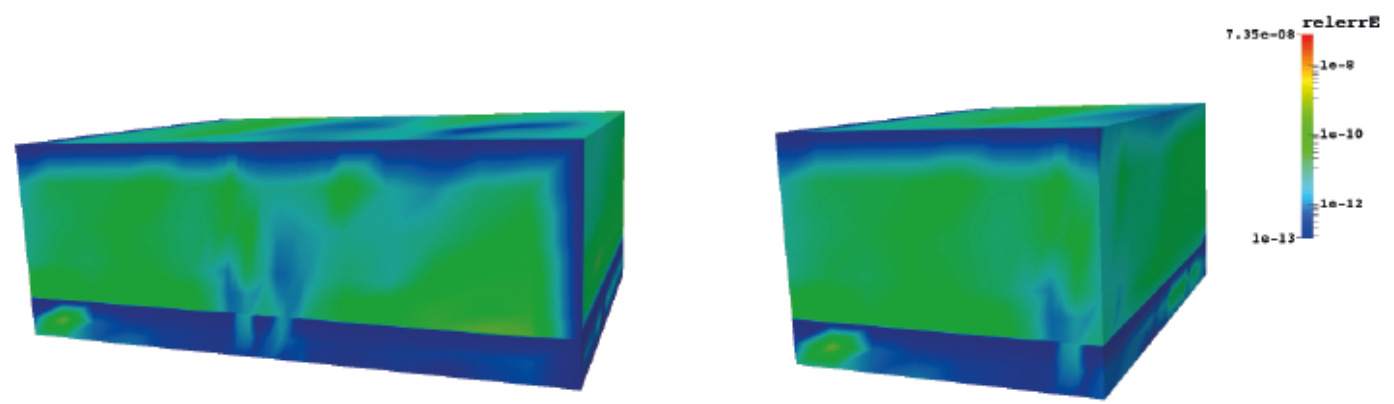

FIG. 7: $\operatorname{err} r_{\mathbb{E}(\boldsymbol{e})}^{r e l}$ for MC of the reduced model, plotted in logarithmic scale. 
systems with a higher number of parameters and, thus, a much larger number of collocation points. The application of collocation combined with MOR to examples with many parameters and more degrees of freedom is future work.

\section{ACKNOWLEDGMENT}

The work reported in this paper was supported by the German Federal Ministry of Education and Research (BMBF), Grant No. 05M10EVA. Responsibility for the contents of this publication rests with the authors.

\section{REFERENCES}

1. Ruehli, A. E., Equivalent circuit models for three-dimensional multiconductor systems, IEEE Trans. Microwave Theory Techniques, 22(3):216-221, 1974.

2. Zaglmayr, S., High order Finite Element Methods for electromagnetic field computation, PhD Thesis, Johannes Kepler Universität Linz, 2006.

3. Bíró, O., Numerische Aspekte von Potentialformulierungen in der Elektrodynamik, Habilitationsschrift, TU Graz, 1992.

4. Monk, P., Finite Element Methods for Maxwell's Equations, Numerical Mathematics and Scientific Computation, Clarendon Press, Oxford University Press, New York, 2003.

5. Matthies, H. G., Uncertainty quantification with stochastic finite elements, In Encyclopedia of Computational Mechanics, Wiley, Hoboken, NJ, 2007.

6. Schwab, C. and Gittelson, C. J., Sparse tensor discretizations of high-dimensional parametric and stochastic PDEs, Acta Numer., 20:291-467, 2011.

7. Ghanem, R. G. and Spanos, P. D., Stochastic Finite Elements: A Spectral Approach, Springer, New York, 1991.

8. Mooney, C. Z., Monte Carlo Simulation, 116 ed., Quantitative Applications in the Social Siences, SAGE University Paper, 1997.

9. Hammersley, J. M. and Handscomb, D. C., Monte Carlo Methods, Methuen, 1964.

10. Babuska, I., Nobile, F., and Tempone, R., A stochastic collocation method for elliptic partial differential equations with random input data, SIAM Rev., 52(2):317-355, 2010.

11. Bagci, H., Yücel, A. C., Hesthaven, J. S., and Michielssen, E., A fast stroud-based collocation method for statistically characterizing EMI/EMC phenomena on complex platforms, IEEE Trans. Electromagn. Compat., 51(2):301-311, 2009.

12. Liu, M., Gau, Z., and Hesthaven, J. S., Adaptive sparse grid algorithms with applications to electromagnetic scattering under uncertainty, Appl. Numer. Math., 61:24-37, 2011.

13. Xiu, D. and Hesthaven, J. S., High-order collocation methods for differential equations with random inputs, SIAM J. Sci. Comput., 27(3):1118-1139, 2005.

14. Benner, P., Gugercin, S., and Willcox, K., A survey of model reduction methods for parametric systems, Preprint MPIMD/1314, Max Planck Institute Magdeburg, August 2013, http://www. mpi-magdeburg.mpg.de/preprints/.

15. Quarteroni, A. and Rozza, G. (Eds.), Reduced Order Methods for Modeling and Computational Reduction, MS \& A Series, Springer, New York, 2014.

16. Lumley, J. L., The structure of inhomogeneous turbulent flows, In: Atmospheric Turbulence and Radio Propagation, Tatarsky, V. I. and Yaglom, A. M. (Eds.), Publishing House Nauka, pp. 166-178, 1967.

17. Sirovich, L., Turbulence and the dynamics of coherent structures. Parts I-III, Quart. Appl. Math., 45(3):561-590, 1987.

18. Volkwein, S., Optimal and suboptimal control of partial differential equations: Augmented Lagrange-SQP methods and reduced-order modeling with proper orthogonal decomposition, Grazer Mathematische Berichte 343, Karl-FranzensUniversität Graz, 2001.

19. Kahlbacher, M. and Volkwein, S., Galerkin proper orthogonal decomposition methods for parameter dependent elliptic systems, Discuss. Math. Diff. Inclus. Control Optim., 27:95-117, 2007.

20. Buffa, A., Maday, Y., Patera, A. T., Prud'homme, C., and Turinici, G., A priori convergence of the Greedy algorithm for the parametrized reduced basis, Math. Model. Numer. Anal., 46(3):595-603, 2012.

Volume 5, Number 3, 2015 
21. Haasdonk, B. and Ohlberger, M., Reduced basis method for finite volume approximations of parametrized linear evolution equations, Math. Model. Numer. Anal., 42(2):277-302, 2008.

22. Haasdonk, B. and Ohlberger, M., Efficient reduced models and a-posteriori error estimation for parametrized dynamical systems by offline/online decomposition, Math. Comput. Model. Dyn. Syst., 17(2):145-161, 2011.

23. Stroud, A. H., Remarks on the disposition of points in numerical integration formulas, Math. Tables Other Aids Comput., 11(60):257-261, 1957.

24. Bungartz, H.-J. and Griebel, M., Sparse grids, Acta Numer., 13:147-269, 2004.

25. Liu, Y., Pileggi, L., and Strojwas, A., Model order reduction of RC(L) interconnect including variational analysis, In Proc. Design Automation Conference, pp. 201-206, 1999.

26. Pulch, R., ter Maten, E. J. W., and Augustin, F., Sensitivity analysis and model order reduction for random linear dynamical systems, Preprint BUW-IMACM 13/07, 2013.

27. Elma, H. C. and Liao, Q., Reduced basis collocation methods for partial differential equations with random coefficients, SIAM/ASA J. Uncert. Quantif., 1(1):192-217, 2013.

28. http://www.modelreduction.org.

29. Papoulis, A., Probability, Random Variables, and Stochastic Processes, Mcgraw-Hill Series in Electrical Engineering, Communications and Signal Processing, Mcgraw-Hill College, 3 ed., McGrou, 1991.

30. Stroud, A. H., Approximate Calculation of Multiple Integrals, Prentice Hall, Englewood Cliff, 1971.

31. Petras, K., Smolyak cubature of given polynomial degree with few nodes for increasing dimension, Numer. Math., 93(4):729753, 2003.

32. Smolyak, S., Quadrature and interpolation formulas for tensor products of certain classes of functions, Soviet Math. Dokl., 4:240-243, 1963.

33. Genz, A. and Keister, B. D., Fully symmetric interpolatory rules for multiple integrals over infinite regions with Gaussian weight, J. Computat. Appl. Math., 71:299-309, 1996.

34. Horwood, J., Aragon, N., and Poore, A., Edgeworth filters for space surveillance tracking, Proc. of Advanced Maui Optical and Space Surveillance Technologies Conference, Ryan, S. (Ed.), 2010.

35. Olver, F. W. J., Quadrature: Gauss-Hermite formula, In: NIST Handbook of Mathematical Functions, Olver, F. W. J., Lozier, D. M., Boisvert, R. F., and Clark, C. W. (Eds.), Cambridge University Press, 2010.

36. Gerstner, T. and Griebel, M., Numerical integration using sparse grids, Numer. Algorithms, 18(3-4):209-232, 1998.

37. Patterson, T. N. L., The optimum addition of points to quadrature formulae, Math. Comput., 22(104):847-856, s21-s31, 1968.

38. Golub, G. H. and Loan, C. F. V., Matrix Computations, Studies in the Mathematical Sciences, 4th ed., Johns Hopkins University, 2012.

39. Ern, A. and Guermond, J.-L., Theory and Practice of Finite Elements, Vol. 159 of Applied Mathematical Sciences, Springer, Berlin, 2004.

40. http://fenicsproject.org/.

41. Logg, A., Mardal, K.-A., and Wells, G. N. (Eds.), Automated Solution of Differential Equations by the Finite Element MethodThe FEniCS Book, Vol. 84 of Lecture Notes in Computational Science and Engineering, Springer-Verlag, Berlin, 2012.

42. Nédélec, J.-C., Mixed finite elements in R3, Numer. Math., 35:315-341, 1980.

43. Nédélec, J.-C., A new family of mixed finite elements in R3, Numer. Math., 50:57-81, 1986.

44. Buffa, A., Trace theorems on non-smooth boundaries for functional spaces related to Maxwell equations: An overview, In: Computational Electromagnetics, Monk, P., Carstensen, C., Funken, S., Hackbusch, W., and Hoppe, R. H. W. (Eds.), Vol. 28, pp. 23-34. Springer-Verlag, Berlin, 2003.

45. Davis, T. A., Direct Methods for Sparse Linear Systems, Fundamentals of Algorithms, SIAM, 2006.

46. Burkardt, J., SGMGA-Sparse grid mixed growth anisotropic rules, http://people.sc.fsu.edu/jourkardt/m_src/sgmga/ sgmga.html. 